\title{
Coulomb Corrections for Interferometry Analysis of Expanding Hadron Systems
}

\author{
Yu.M. Sinyukov*, R. Lednicky $\dagger$ J. Pluta ${ }^{\ddagger}$, B. Erazmus
}

SUBATECH, (UMR, Universite, Ecole des Mines, IN2P3/CNRS), 4 rue Alfred Castler, La Chantrerie, BP 20722. 44307 NANTES CEDEX 3, France.

\author{
S.V. Akkelin
}

ITP Kiev 252143, Metrologicheskaya 14b,Ukraine.

化 September 12, 1997

\begin{abstract}
The problem of the Coulomb corrections to the two-boson correlation functions for the systems formed in ultra-relativistic heavy ion collisions is considered for large effective system volumes. The modification of the standard zero-distance correction (so called Gamow or Coulomb factor) has been proposed for such a kind of systems. For the $\pi^{+} \pi^{+}$and $K^{+} K^{+}$correlation functions the analytical calculations of the Coulomb correction are compared with the exact numerical results.
\end{abstract}

${ }^{*}$ Permanent address: Institute for Theoretical Physics, Kiev 252143, Metrologicheskaya 14b, Ukraine.

tPermanent address: Institute of Physics, Na Slovance 2, 18040 Prague 8, Czech Republic.

${ }^{\ddagger}$ Permanent address: Institute of Physics, Warsaw University of Technology, Koszykowa 75, 00-662 Warsaw, Poland. 


\section{Introduction}

One of the main goals of the experiments with ultra-relativistic heavy ions is a possibility to study the properties of the hadronic matter at very high temperatures and energy densities. There is a direct connection between these properties and space-time evolution of the systems created in nuclear collisions. The boson interferometry method [1],[2] allows to extract the important features of the system evolution. The interferometry radii resulting from a Gaussian fit of the two-boson correlation function reflect the space-time structure of a source. For thermalized expanding systems the interferometric radii at high $p_{T}$ are associated directly with the system's lengths of homogeneity $\lambda$ [3]. The latter contain the basic parameters of the evolution, such as proper time of the expansion, transverse radius, intensity of transversal flows, freeze-out temperature, etc. To extract these parameters one needs to measure the interferometry radii in different regions of $y$ and $p_{T}$ [4].

The real experimental situation with the interferometry analysis is complicated because of the effects of the strong and Coulomb final state interactions (FSI). In heavy ion collisions the effect of the short-range strong interaction is usually small and can be neglected. On the contrary, the effect of the Coulomb FSI dominates the behavior of the spectra of two charged particles at very small relative momenta. Usually it is taken into account with the help of the standard Coulomb factor (the modulus squared of the non-relativistic Coulomb wave function at zero distance). This procedure is well founded if the effective size of the emitting source is much smaller than the Bohr radius $|a|$ and smaller or compatible with the inverse relative momentum. At the same time, the experimental behavior of the effective sizes in $A+A$ collisions still well follows the $R \sim(d N / d y)^{1 / 3}$ tendency [5]. It means that for current experiments with most heavy nuclei such as lead and for future experiments at $R H I C$ and $L H C$ the average distances $r^{*}$ between any two emitted particles in their c.m.s. will be larger, Coulomb interaction will be weaker and so the simple Gamow correction will be too strong and will have to be modified (hereafter the quantities related to the two-particle c.m.s. are marked by the asterisk).

It can be seen that the Coulomb correction generally depends on the momentum region where the pairs are registered. To understand this, note that the distance $r^{*}$ between two emitted particles is considered for the Coulomb problem in the c.m.s. of the pair of two interfering particles with similar 
momenta. If, for example, we select particle pairs emitted perpendicular to the reaction axis by a static source of the size $R$, the corresponding size $R^{*}$ in the c.m.s. of the pair will increase with the increasing momentum due to the Lorentz transformation. For hydrodynamically expanding systems there is specific momentum dependence of the homogeneity lengths and the corresponding interferometric radii even in the proper c.m.s. [3], [4].

One of the main aims of the paper is to analyze the Coulomb corrections for the large expanding hadron sources.

\section{Correlations of non-interacting bosons}

The correlation function is usually defined as a ratio of the two-particle spectrum to the product of the one-particle ones:

$$
C\left(p_{1}, p_{2}\right)=\omega_{1} \omega_{2} \frac{d^{6} N}{d^{3} \mathbf{p}_{1} d^{3} \mathbf{p}_{2}} /\left(\omega_{1} \frac{d^{3} N}{d^{3} \mathbf{p}_{1}} \omega_{2} \frac{d^{3} N}{d^{3} \mathbf{p}_{\mathbf{2}}}\right)
$$

where $\omega_{i}=\left(m_{i}^{2}+\mathbf{p}_{i}^{2}\right)^{1 / 2}$ are particle energies. For non-interacting identical spinless bosons we can write [1]

$$
C\left(p_{1}, p_{2}\right)=1+\Lambda\langle\cos (q x)\rangle=\Lambda\left\langle|\psi|^{2}\right\rangle+(1-\Lambda)
$$

where $\psi$ is the symmetrized "relative-coordinate" part of a two-particle wave function $\psi_{p_{1}, p_{2}}\left(x_{1}, x_{2}\right)$,

$$
\begin{aligned}
\psi & =\frac{1}{\sqrt{2}}\left[\psi_{p_{1} p_{2}}(x)+\psi_{p_{2} p_{1}}(x)\right] \\
\psi_{p_{1} p_{2}}(x) & =e^{i q x / 2}, \psi_{p_{1}, p_{2}}\left(x_{1}, x_{2}\right)=e^{2 i p X} \psi_{p_{1} p_{2}}(x)
\end{aligned}
$$

for identical bosons. Here $q=p_{2}-p_{1}, p=\left(p_{1}+p_{2}\right) / 2$, the averaging is done over the relative 4-coordinates $x=x_{1}-x_{2}$ and $X=\left(x_{1}+x_{2}\right) / 2$ of the emission points of those bosons which can give an observable interference effect. The corresponding fraction of such bosons is taking into account by the so-called chaoticity parameter $\Lambda \leq 1$. For non-identical particles $\psi=\psi_{p_{1} p_{2}}(x)$ and from the right part of Eq.(2) follows $C\left(p_{1}, p_{2}\right)=1$. 
Assuming that particles are emitted from the narrow enough $4 \mathrm{D}$ volume, one can explore the idea of freeze-out hypersurface $\sigma$ and expresses the corresponding average in (2) by the Wigner function at $\sigma$ [3]

$$
\langle\cos (q x)\rangle \cong \frac{\int d^{3} \sigma_{\mu}\left(x_{1}\right) d^{3} \sigma_{\nu}\left(x_{2}\right) p^{\mu} p^{\nu} f_{W}\left(x_{1}, p\right) f_{W}\left(x_{2}, p\right) \cos (q x)}{\int d^{3} \sigma_{\mu}\left(x_{1}\right) d^{3} \sigma_{\nu}\left(x_{2}\right) p^{\mu} p^{\nu} f_{W}\left(x_{1}, p\right) f_{W}\left(x_{2}, p\right)}
$$

The approximation correspond to $p_{1} \cong p_{2} \cong p$ (smoothness condition) in the denominator.

In the so called longitudinally co-moving system (LCMS) in which the boson pair is emitted transverse to the collision axis $\left(p_{L}=0\right)$ the interferometric radii $R_{j}$ are determined according to a simple Gaussian parametrization:

$$
C\left(p_{1}, p_{2}\right)=1+\Lambda \exp \left(-q_{L}^{2} R_{L}^{2}-q_{O}^{2} R_{O}^{2}-q_{S}^{2} R_{S}^{2}\right),
$$

where the components of the vector $\mathbf{q}$ are chosen parallel to the collision axis $(\mathrm{z}=\mathrm{L})$, parallel to the vector $\mathbf{p}_{T}(\mathrm{x}=\mathrm{O})$ and perpendicular to the production plane $(z, x)$ of the pair $(y=S)$.

As a realistic example, consider a cylindrically symmetric radiating system, with a Gaussian transverse radius $\bar{R}_{T}$, longitudinal quasi-inertial flow at the final stage [6], [7]: $r_{L} / t=\tanh y_{L}$, and expanding in radial direction $\hat{\mathbf{r}}_{T}=\mathbf{r}_{T} / r$ linearly in transversal rapidity $y_{T}$ at small $r_{T}$. It decays at the freeze-out temperature $T=1 / \beta$ on the freeze-out hypersurface $\sigma: \tau=\sqrt{t^{2}-r_{L}^{2}} \cong$ const. For such a kind of system, we can use the Wigner function in statistical (hydrodynamic) approach, i.e. put, in Boltzmann approximation

$$
f_{W}(p, x) \propto \exp \left(-\beta p^{\mu} u_{\mu}(x)\right) \exp \left(-\mathbf{r}_{T}^{2} /\left(2 \bar{R}_{T}^{2}\right)\right) .
$$

In the framework of the saddle-point method at $\beta m_{T} \gg 1, m_{T}=\sqrt{m^{2}+p_{T}^{2}}$ we can use for the distribution of the relative coordinates $\mathbf{r}=\mathbf{x}_{1}-\mathbf{x}_{2}$ of the emission points in the LCMS the simple expression:

$$
f_{W}\left(p, x_{1}\right) \cdot f_{W}\left(p, x_{2}\right) \propto \exp \left(-r_{x}^{2} /\left(4 R_{O}^{2}\right)-r_{y}^{2} /\left(4 R_{S}^{2}\right)-r_{z}^{2} /\left(4 R_{L}^{2}\right)\right)
$$

The radii $R_{j}$ are related to the homogeneity lengths $\lambda_{j}$ of the system:

$$
R_{j}^{-2}=2\left(\lambda_{j}(\bar{x})\right)^{-2}=\left|\frac{\partial^{2} f_{W}(p, \bar{x})}{\partial^{2} x_{j}}\right|\left(f_{W}(p, \bar{x})\right)^{-1}
$$


at the saddle-points $\bar{x}(p)$ and equals to [4]:

$$
\begin{aligned}
& R_{L}^{2}=\frac{\tau^{2}}{\beta m_{T}}\left(1-\bar{v}_{T}^{2}\right)^{\frac{1}{2}}, R_{O}^{2}=\frac{\bar{R}_{v}^{2}}{\beta m_{T}+\alpha}\left(1-\bar{v}_{T}^{2}\right)^{-\frac{1}{2}} \\
& R_{S}^{2}=\frac{\bar{R}_{v}^{2}}{4 \beta p_{T} \bar{v}_{T}}\left(1-\bar{v}_{T}^{2}\right)^{\frac{1}{2}} \ln ^{2}\left(\left(1+\bar{v}_{T}\right) /\left(1-\bar{v}_{T}\right)\right)
\end{aligned}
$$

Here the parameter $\alpha=\bar{R}_{v}^{2} / \bar{R}_{T}^{2}$ describes the intensity of transversal flow. It is equal to the ratio squared of the transverse hydrodynamic length $\bar{R}_{v}$ (near the collision axis) to the Gaussian-like transverse radius $\bar{R}_{T}$ of a decaying system. The transverse hydrodynamic velocity at the saddle-point is

$$
\bar{v}_{T} \equiv \tanh y_{T}(\bar{x}(p))=\frac{\beta p_{T}}{\beta m_{T}+\alpha} .
$$

For very intensive relativistic transverse flows, $\alpha \rightarrow 0$. On the other hand for $\alpha \gg 1$ we have non-relativistic transverse flows and for $\alpha=\infty$ - the $1 D$-expansion in longitudinal direction (transverse flows are absent). In the latter case Eqs. (9) recover the results of the papers $[8,9]$ at large $m_{T}$. In particular, $R_{L}=\tau \sqrt{T / m_{T}}$ and $R_{O}=R_{S}=\bar{R}_{T}$.

It should be noted that the direct application of this model to pion correlations is complicated by a strong resonance contribution which is increasing with decreasing $p_{T}$. Nevertheless, just for demonstration aims we have applied the asymptotic (large- $m_{T}$ ) formulas (9) to the NA49 data on pion interferometric radii in $\mathrm{Pb}+\mathrm{Pb}$ collisions measured at midrapidity in different $p_{T}$ regions [10]. A good agreement is found for $\alpha=1, T=m_{\pi}$ and $\tau=\bar{R}_{T}=8.6 \mathrm{fm}$. Below we will use these parameters of the system evolution in a study of the Coulomb corrections to the kaon correlations as well. For $\mathrm{LHC}$ energies we will use the average transverse radius of system proper time $\tau=\bar{R}_{T}=18 \mathrm{fm}$.

\section{Finite-size Coulomb corrections}

The problem of the correlations of the particles with a non-negligible final state interaction is rather complex and practically can be solved only on the conditions of a small phase-space density of the emitted particles and sufficient smoothness of the one-particle amplitudes as compared with 
a sharp $q$-dependence of the two-particle one due to the joint effect of quantum statistics and FSI. On the small phase-space density, the two particles with a small relative momentum can be considered in isolation of the rest of the system and the effect of FSI reduces to the substitution of the free plane wave amplitudes (3) by the non-symmetrized Bethe-Salpeter amplitudes in the continuous spectrum of the two-particle states [11, 12, 13]: $\psi_{p_{1}, p_{2}}\left(x_{1}, x_{2}\right) \rightarrow \psi_{p_{1}, p_{2}}^{B S}\left(x_{1}, x_{2}\right)=e^{2 i p X} \psi_{p_{1} p_{2}}^{B S}(x)$. In other words, the role of the plane waves as a functional basis which the asymptotic two-particle state is projected on is transferred to the Bethe-Salpeter amplitudes (the waves distorted by the interaction) [12].

At equal emission times in the two-particle c.m.s. $\left(t^{*}=0\right)$ the amplitude $\psi_{p_{1} p_{2}}^{B S}(x)$ coincides with a stationary solution of the scattering problem $\psi_{p_{1} p_{2}}^{B S}(x) \rightarrow \psi_{-\mathbf{k}^{*}}\left(\mathbf{r}^{*}\right)$. Note that in the two-particle c.m.s. $\mathbf{p}_{1}^{*}=-\mathbf{p}_{2}^{*}=\mathbf{k}^{*}$, $\mathbf{q}^{*}=-2 \mathbf{k}^{*}$ and, for equal-mass particles, $q_{0}^{*}=0$. The function $\psi_{-\mathbf{k}^{*}}\left(\mathbf{r}^{*}\right)$ is $[13,14]$

$$
\psi_{-\mathbf{k}^{*}}\left(\mathbf{r}^{*}\right) \rightarrow \mathrm{e}^{i \delta_{0}^{c}} \sqrt{A_{c}(\eta)}\left[\mathrm{e}^{-i \mathbf{k}^{*} \mathbf{r}^{*}} F(-i \eta, 1, i \xi)+f_{c}\left(k^{*}\right) \tilde{G}(\rho, \eta) / r^{*}\right],
$$

where $\rho=k^{*} r^{*}, \xi=\mathbf{k}^{*} \mathbf{r}^{*}+k^{*} r^{*} \equiv \rho\left(1+\cos \theta^{*}\right), \eta=1 /\left(k^{*} a\right), a=1 / \mu z_{1} z_{2} e^{2}$ is the Bohr radius ( $\mu$ is the reduced mass of the two particles, $z_{1} z_{2} e^{2}$ is the product of the particle electric charges $), \delta_{0}^{c}=\arg \Gamma(1+i \eta)$ is the Coulomb s-wave phase shift, $f_{c}\left(k^{*}\right)=\left(\mathrm{e}^{2 i \delta_{0}}-1\right) /\left(2 i k^{*} A_{c}\right)$ is the s-wave scattering amplitude due to the strong interaction renormalized by the Coulomb force,

$$
A_{c}(\eta)=2 \pi \eta[\exp (2 \pi \eta)-1]^{-1}
$$

is the Coulomb factor - modulus squared of the non-relativistic Coulomb wave function at zero distance - introduced already by Fermi in his theory of $\beta$-decay,

$$
F(\alpha, 1, z)=1+\alpha z+\alpha(\alpha+1) z^{2} / 2 !^{2}+\alpha(\alpha+1)(\alpha+2) z^{3} / 3 !^{2}+\cdots
$$

is the confluent hypergeometric function and $\tilde{G}(\rho, \eta)$ is the combination of the usual singular and regular s-wave Coulomb functions. We will neglect by the strong interaction in further analytical formulas considering only $\pi \pi$ and $K^{+} K^{+}$systems where it does not play essential role in current (and planning) A+A collisions. We will control the distortion that they give on the final result using the strong FSI "switch on" and "switch off" options in corresponding Monte Carlo simulations with computer code of ref. [13]. 
To account for the finite-size corrections, we could introduce a generalized size-dependent Coulomb factor as an average modulus squared of the nonsymmetrized Coulomb wave function:

$$
\tilde{A}_{c}=\left\langle\left|\psi_{-\mathrm{k}^{*}}^{c}\left(\mathbf{r}^{*}\right)\right|^{2}\right\rangle
$$

The correlation function for unlike sign bosons follows from the right part of Eq. (2) with substitution $\psi=\psi_{p_{1} p_{2}}(x) \rightarrow \psi_{-\mathbf{k}^{*}}\left(\mathbf{r}^{*}\right)$ :

$$
C_{+-}\left(p_{1}, p_{2}\right)=\Lambda_{+-} \tilde{A}_{c}^{+-}+\left(1-\Lambda_{+-}\right) .
$$

In the same way one can easily find the form of the correlation function of identical bosons. We can see that the another size-dependent Coulomb factor $\widetilde{B}_{c} \neq \widetilde{A}_{c}$ appears due to the effect of symmetrization,

$$
\begin{aligned}
C_{++}\left(p_{1}, p_{2}\right) & =\Lambda_{++}\left[\tilde{A}_{c}^{++}+\tilde{B}_{c}^{++}\langle\cos (q x)\rangle\right]+\left(1-\Lambda_{++}\right), \\
\tilde{B}_{c}^{++} & =\left\langle\operatorname{Re}\left[\psi_{-\mathbf{k}^{*}}^{c}\left(\mathbf{r}^{*}\right) \psi_{\mathbf{k}^{*}}^{c *}\left(\mathbf{r}^{*}\right)\right]\right\rangle /\langle\cos (q x)\rangle .
\end{aligned}
$$

The Coulomb factor $\widetilde{B}_{c}$ on the condition $\left\langle r^{*}\right\rangle /|a| \ll 1$ has to coincide with $\widetilde{A}_{c}$ at $k^{*}\left\langle r^{*}\right\rangle \ll 1$. In the limit $\left\langle r^{*}\right\rangle /|a| \rightarrow 0$ they are both equal to the Coulomb factor $A_{c}(\eta)$. Using the expansion of the confluent hypergeometric function $F$ in Eq. (13), in the lowest powers of $\eta \xi, \rho$ we can estimate the deviation $\widetilde{B}_{c}$ from $\tilde{A}_{c}$ in the limit of isotropic Gaussian distribution. At $R^{*} \cong 6.6 \mathrm{fm}$ (SPS energy) we find that at small $k^{*}\left\langle r^{*}\right\rangle \ll 1$ the ratio $\widetilde{B}_{c} / \widetilde{A}_{c}$, for $\pi^{+} \pi^{+}$and $K^{+} K^{+}$systems, is lower than unity by only $\sim 0.1 \%$ and $\sim 1.4 \%$, respectively. At LHC $\left(R^{*} \cong 15 \mathrm{fm}\right)$ we expect $\left\langle r^{*}\right\rangle$ by the factor $(15 / 6.6)^{2}$ larger, corresponding to the increase of these deviations by a factor of 5. Regarding the behavior of the ratio $\widetilde{B}_{c} / \widetilde{A}_{c}$ at large $k^{*}\left\langle r^{*}\right\rangle \gg 1$, due to a rapid vanishing of the interference term $\widetilde{B}_{c}\langle\cos (q x)\rangle \rightarrow 0$, we can harmlessly put this ratio equal to any finite value in this region. We will thus adopt here a simple ansatz

$$
\tilde{B}_{c}\left(k^{*}\right)=\widetilde{A}_{c}\left(k^{*}\right),
$$

It leads, on the absence of the strong interaction, to the following approximation for the correlation function of the identical charged bosons (see Eq. (16)):

$$
C_{++}\left(p_{1}, p_{2}\right)=\Lambda_{++} \tilde{A}_{c}^{++} C_{Q S}\left(p_{1}, p_{2}\right)+\left(1-\Lambda_{++}\right) .
$$


However this ansatz could introduce the distortion $\sim\left\langle r^{*}\right\rangle / a$ in the fitted interferometric radii squared. We will estimate it comparing the corresponding analytical calculations with the exact numerical ones.

The size-dependent Coulomb factor $\tilde{A}_{c}$ in the limit of the very small momenta, $1 /|\eta|=k^{*}|a| \ll 1$, can be obtained in the main approximation by the $1 /|\eta|$ - expansion in series of the confluent hypergeometric function in (11):

$$
\begin{gathered}
\tilde{A}_{c} \cong A_{c}(\eta)\left\langle\left|J_{0}(2 i \sqrt{\eta \xi})\right|^{2}\right\rangle \rightarrow \\
A_{c}(\eta)\left[1+2\left\langle r^{*}\left(1+\cos \theta^{*}\right)\right\rangle / a+3\left\langle r^{* 2}\left(1+\cos \theta^{*}\right)^{2}\right\rangle / 2 a^{2}\right],
\end{gathered}
$$

where $J_{0}$ is the usual Bessel function. The arrow in Eq. (20) indicates the limit of $\left\langle r^{*}\right\rangle /|a| \ll 1$ we are interested $(|a|=388 \mathrm{fm}$ for pions and $111 \mathrm{fm}$ for kaons). In this limit

$$
\tilde{A}_{c}=A_{c}(\eta)\left[1+2\left\langle r^{*}\right\rangle / a+3 \pi\left\langle r^{*}\right\rangle^{2} / 4 a^{2}\right] .
$$

We include here $\left\langle r^{*}\right\rangle^{2} / a^{2}$ - term calculated in symmetrical Gaussian approach to apply this result to kaon interferometry at RHIC and LHC.

In order to consider much more wide region $|\eta|=k^{*}|a| \gg 1$ let us reexpand the confluent hypergeometric function over small parameter $|\eta|=$ $1 /|a| k^{*} \ll 1$ and keep only linear terms. Then

$$
F(-i \eta, 1, i \xi) \cong 1+i \eta \operatorname{Ein}(-i \xi)
$$

where [15]

$$
\operatorname{Ein}(-z)=-\sum_{n=1}^{\infty} \frac{z^{n}}{n n !}
$$

In this approach

$$
\tilde{A}_{c} \cong A_{c}(\eta)\left[1+2 \widetilde{r}\left(k^{*}\right) / a\right] \text { and } \tilde{r}\left(k^{*}\right)=\langle\operatorname{Si}(\xi)\rangle / k^{*},
$$

where [15]

$$
S i(\xi)=\frac{1}{2 i}[\operatorname{Ein}(i \xi)-\operatorname{Ein}(-i \xi)]=\xi-\frac{\xi^{3}}{3 \cdot 3 !}+\cdots
$$


Let us point that for $k^{*}|a| \gg 1(24)$ is correct for all $\left\langle r^{*}\right\rangle /|a|$. Compare (24) with (21) we see that (24) is correct also for $k^{*}|a| \ll 1$ at the linear approximation in $\left\langle r^{*}\right\rangle /|a|$ provided that $\left\langle r^{*}\right\rangle /|a| \ll 1$. Finally we can conclude that Eq. (24) is correct approximation for $\widetilde{r}\left(k^{*}\right) /|a| \ll 1$. Neglection of the $\widetilde{r}\left(k^{*}\right) / a$ term corresponds to so called Gamow-factor approach.

It may be interesting to look how the quantum Coulomb factor in Eq. (24) reproduces the quasi-classical behavior at $k^{*} r^{*} \gg 1$. For this, we will follow ref. [16] and assume spherically symmetric distribution of the relative coordinates $\mathbf{r}^{*}$. Then the integral over the angular coordinates can be done explicitly and we get ${ }^{1}$ :

$$
\begin{gathered}
\tilde{A}_{c}=A_{c}(\eta)\left[1+2\left\langle\frac{\operatorname{Si}\left(2 k^{*} r^{*}\right)}{k^{*} a}\right\rangle-\left\langle\frac{1-\cos \left(2 k^{*} r^{*}\right)}{r^{*} a k^{* 2}}\right\rangle\right] \\
\rightarrow A_{c}(\eta)\left[1+\pi /\left(k^{*} a\right)-\left\langle\left(r^{*} a k^{* 2}\right)^{-1}\right\rangle_{k^{*}}\right] \rightarrow 1-\left\langle\left(r^{*} a k^{* 2}\right)^{-1}\right\rangle_{k^{*}},
\end{gathered}
$$

where the arrows indicate the limit $k^{*}\left\langle r^{*}\right\rangle \gg 1$. The second arrow corresponds to the substitution $A_{c} \rightarrow 1-\pi /\left(a k^{*}\right)$ which immediately leads to the quasi-classical formula [18] in the first order in $a^{-1}$.

Compared to the large- $k^{*}$ behavior of the zero-distance Coulomb factor $A_{c} \rightarrow 1-\pi /\left(a k^{*}\right)$, the difference from unity of the size-dependent Coulomb factor vanishes much faster: $\widetilde{A}_{c} \rightarrow 1-\left\langle\left(r^{*} a k^{* 2}\right)^{-1}\right\rangle_{k^{*}}$. The long $k^{*}$-tail of the simple factor $A_{c}$, in fact, revealed itself in the corresponding false tail observed in the "Gamow corrected" correlation functions in a number of experiments (see, e.g., [19]).

It should be stressed that the tail of the simple Coulomb factor at $\left.k^{*}\left\langle r^{*}\right\rangle\right\rangle$ 1 has nothing to do with the large particle multiplicities and/or with a rapid expansion of the source in ultra-relativistic nuclear collisions, as suggested in [19]. In fact, the NA35 correlation data for both like and unlike charge

\footnotetext{
${ }^{1}$ Note that the third term in Eq. (26) was lost in a similar integral in ref.[16]. This and also an error in the definition of the term analogous to the factor $\widetilde{B}_{c}$ (forgotten the substitution $\mathbf{k}^{*} \rightarrow-\mathbf{k}^{*}$ in the complex conjugate Coulomb wave function) led to a curious behavior of the ratio $C / C_{Q S} \approx \widetilde{A}_{c}$ for the identical charged pions. For small values of $k^{*}$, contrary to the expansion of the hypergeometrical function $F$ in Eq. (13), this ratio happened to increase with decreasing $\left\langle r^{*}\right\rangle$ and at $\left\langle r^{*}\right\rangle=6 \mathrm{fm}$ exceeded unity by $20 \%$. In fact, it was checked in ref. [17] that at small $k^{*}<10 \mathrm{MeV} / \mathrm{c}$, this ratio, in accordance with Eq. (21), increases linearly with the increasing $\left\langle r^{*}\right\rangle$ up to the values of the order of $10 \mathrm{fm}$ and only slightly exceeds unity (by $3 \%$ at $\left\langle r^{*}\right\rangle=6 \mathrm{fm}$ ).
} 
pion pairs appears to be in a good agreement with the standard two-body FSI calculations accounting for the finite size effect according to Eq. (24).

\section{Analytical approximations to the Coulomb corrections}

First, we will try to tail the behavior of the Coulomb factor $\tilde{A}_{c}\left(k^{*}\right)$ in the quantum region $k^{*}\left\langle r^{*}\right\rangle \ll 1$ (see Eq. (21)) and in the quasi-classical one $k^{*}\left\langle r^{*}\right\rangle \gg 1$ (see Eq. (26). We will do it according to a simple ansatz:

$$
\begin{gathered}
\tilde{A}_{c}\left(k^{*}\right) \cong A_{c}(\eta)\left(1+2 \frac{\left\langle r^{*}\right\rangle}{a}+3 \pi\left\langle r^{*}\right\rangle^{2} / 4 a^{2}\right) \theta\left(\tilde{k}-k^{*}\right)+ \\
\left(1-\frac{d}{a\left\langle r^{*}\right\rangle k^{* 2}}\right) \theta\left(k^{*}-\tilde{k}\right) .
\end{gathered}
$$

This ansatz contains three parameters $\left\langle r^{*}\right\rangle, \tilde{k}$ and $d$, but only one of them $\left(\left\langle r^{*}\right\rangle\right)$ can be considered as free due to the tailing conditions which demand the smoothness of the function and its derivation at the point $\widetilde{k}$. It give us

$$
\tilde{k}\left\langle r^{*}\right\rangle \cong(\pi / 4)\left(1+2\left\langle r^{*}\right\rangle / a\right)
$$

and

$$
d=a\left\langle r^{*}\right\rangle \widetilde{k}^{2}\left[1-A_{c}(\eta)\left(1+2 \frac{\left\langle r^{*}\right\rangle}{a}+3 \pi\left\langle r^{*}\right\rangle^{2} / 4 a^{2}\right)\right],
$$

Using this one-parameter fit it is possible to find the average relative coordinate $\left\langle r^{*}\right\rangle$ using even unlike sign charge pions correlation function. As far as the interferometry of identical bosons is concerned the fit which is based on the Gaussian-like distribution similar to (7) that used for hydrodynamic approach has the form

$$
C\left(p_{T}, q\right)=\Lambda \tilde{A}_{c}\left(k^{*}\right)\left[1+\exp \left(-q_{O}^{2} R_{O}^{2}\left(p_{T}\right)-q_{S}^{2} R_{S}^{2}\left(p_{T}\right)-q_{L}^{2} R_{L}^{2}\left(p_{T}\right)\right]+1-\Lambda\right.
$$

where $k^{*}=\left(q_{O}^{2}+q_{S}^{2}+q_{L}^{2}-q_{0}^{2}\right)^{1 / 2} / 2$ and $\left\langle r^{*}\right\rangle$ can to be expressed analytically in the case of practical interest, when $R_{S} \cong R_{L} \cong R$ and $R_{O}^{*}=\frac{m_{T}}{m} R_{O} \geq R$. 
Then (arrow means symmetric case $R_{O}^{*}=R$ )

$$
\left\langle r^{*}\right\rangle=\frac{2}{\sqrt{\pi}} R_{O}^{*}\left[1+\left(1-\epsilon^{2}\right) \frac{1}{2 \epsilon} \ln \frac{1+\epsilon}{1-\epsilon}\right] \rightarrow \frac{4}{\sqrt{\pi}} R, \epsilon=\left(1-\left(R / R_{O}^{*}\right)^{2}\right)^{1 / 2}
$$

Note, that the combined correction fit (30) does not contain any new parameters, only standard interferometry radii. The result of fitting demonstrated at Fig. 1 for $K^{+} K^{+}$correlation function calculated with the help of the Monte Carlo simulation in RQMD (v.1.08) with FSI code. We are demonstrating at the plots also standard Gamow approach to see obviously the degree of a finite size effect for different systems. For hydrodynamic model the radii are depending on $p_{T}$ according to Eq.(9). We can conclude that our method based on the simple factorization of the complete correlation functions works as well as the complicated methods of the Refs. [20].

If the source is essentially non-Gaussian (in hydrodynamic approach it can be the case for pions at small $p_{T}$ due to relatively small masses and resonance contributions) it will be good to go beyond the Gaussian approach to solve the Coulomb problem. Note, that in the considered limit $\tilde{r}\left(k^{*}\right) /|a| \ll 1$ the ratio $\tilde{A}_{c}\left(k^{*}\right) / A_{c}(\eta)$ depends on the nature of the two-particle system practically only through the linear combination of the inverse pair Bohr "radius" $a$. Since $a= \pm|a|$ for the like and unlike charge bosons, respectively, we can then relate, on certain conditions, the correlation functions $C_{++}$and $C_{+-}$. Thus, assuming that there is no difference in the production of the positive and negative bosons of the same type and neglecting the effect of the strong FSI, we can use Eqs. (15), (19) and (24) to get at $\Lambda=1$, i.e. in the absence of the long-lived sources and the sources of the "coherent" bosons, the following equality: expression for the correlation function $C_{Q S}\left(p_{1}, p_{2}\right)=1+\langle\cos (q x)\rangle$ due to the QS only:

$$
C_{Q S}\left(p_{1}, p_{2}\right) \cong \frac{C_{++}\left(p_{1}, p_{2}\right)}{A_{c}\left(\eta_{++}\right)}\left[2-\frac{C_{+-}\left(p_{1}, p_{2}\right)}{A_{c}\left(\eta_{+-}\right)}\right]^{-1} .
$$

With the considered accuracy, accounting for the terms linear in $\tilde{r}\left(k^{*}\right) / a$, we can also write, just by multiplying Eqs. (15) and (16) at $\Lambda=1$, an alternative expression for $C_{Q S}$ valid on the same conditions as Eq. (32):

$$
C_{Q S}\left(p_{1}, p_{2}\right) \cong C_{++}\left(p_{1}, p_{2}\right) C_{+-}\left(p_{1}, p_{2}\right)\left[A_{c}\left(\eta_{++}\right) A_{c}\left(\eta_{+-}\right)\right]^{-1}
$$


The latter expression can be compared with the intuitive prescription recently suggested by the NA35 collaboration [19]. This prescription coincides with Eq. (33) up to the inverse product of the Coulomb factors and thus it explodes at $k^{*}<2 \pi /|a|$ : Fig.1, $(\pi \pi)$. The methods based on Eq.(32) (or (33)) does not work for kaons because the strong short-range interaction for system $K^{+} K^{-}$: see Fig1, $(K K)$.

In Fig.2a we demonstrate the validity of Eq. (32) for the pion pairs simulated using the RQMD model for $P b+P b$ collisions at SPS. To follow as closely as possible the applicability conditions of this equation, we have excluded the pions from the $K_{s}^{0}$ - and $\Lambda$-decays and also - switched off the strong FSI at Fig 2a. The maximal deviation is $1.5-2 \%$. The same is true for the NA35 prescription only at $q_{i n v} \equiv 2 k^{*}>10 \mathrm{MeV}$. In the situation when the strong FSI switch on (Fig. 2b) the corresponding deviation is $3-4 \%$. At LHC the effect of the strong FSI between the pions will be by a factor of $2 \div 3$ smaller due to the increased effective size.

In the Fig.3 we compare the both our analytical approaches (30) and (32) for $\pi^{+} \pi^{+}$-interferometry at SPS and LHC- energies. The corresponding parameters are fixed in Section 2: $\tau=\bar{R}_{T}=8.6 \mathrm{fm}$ for $\mathrm{Pb}+\mathrm{Pb}$ SPS and $\tau=$ $\bar{R}_{T}=18 \mathrm{fm}$ LHC energies. The $p_{T}$ dependance of the length of homogeneity is defined as (9). As we see it is good enough even for LHC. For SPS Pb+PB collision the effective sizes of the system in the c.m.s. of pairs are for $p_{T}=$ $0.15 \mathrm{GeV}: R_{O}^{*}=R=7.2 \mathrm{fm},\left\langle r^{*}\right\rangle=16.2 \mathrm{fm}$; for $p_{T}=0.4 \mathrm{GeV}: R_{O}^{*}=16.7$ $\mathrm{fm}, R=4.3 \mathrm{fm},\left\langle r^{*}\right\rangle=21.4 \mathrm{fm}$. The corresponding sizes are 2.1 larger for LHC energies. The maximal deviation is $1 .-1.5 \%$ for SPS and $3 \%$ (combine correction $), 5 \%\left(C^{++} \backslash C^{+-}\right.$-correction) for very asymmetric source at large $p_{T}=0.4$.

The effect of the strong FSI is much more important for kaons. This is demonstrated in Fig. 1, $(K K)$. Regarding the NA35 prescription, even in the absence of the strong interaction, it could now be applied (due to the Bohr radius is smaller by a factor of 3.5) only at $q_{\text {inv }}>35 \mathrm{MeV}$. Note that combined correction deals only with $K^{+} K^{+}$where the strong FSI is weak enough and so work good. The another reason for this is that due to relatively large masses the effective source for kaons is actually Gaussianlike in dynamical models (e.g., in the hydrodynamic approach, see [4]) that is adequate to the combined correction method. For kaons one can neglect of a resonance contributions and therefore can use the Wigner function in statistical (hydrodynamic) approach. 
The results presented at the Fig. 4 demonstrates the good agreement within a few percents of the numerical calculations with our analytical results (30), (32). The results for $K^{+} K^{+}$-Coulomb correction at $p_{T}=0.15 \mathrm{GeV}$ and $p_{T}=0.40 \mathrm{GeV}$ are calculated for the same system's parameters which was used for the $\pi^{+} \pi^{+}$interferometry at $P b+P b 158 \mathrm{AGeV}$ [10]. For SPS $\mathrm{Pb}+\mathrm{Pb}$ collision the effective sizes of the system in the c.m.s. of pairs are for $p_{T}=0.15 \mathrm{GeV}: R_{O}^{*}=R=4.3 \mathrm{fm},\left\langle r^{*}\right\rangle=9.8 \mathrm{fm}$; for $p_{T}=0.4 \mathrm{GeV}$ : $R_{O}^{*}=5.5 \mathrm{fm}, R=4.4 \mathrm{fm},\left\langle r^{*}\right\rangle=9.8 \mathrm{fm}$. The corresponding sizes are in 2.1 times larger for LHC energies. To demonstrate the region of the applicability of our method we demonstrate at Fig. $4 \mathrm{~d}$ the comparison at $p_{T}=0.8 \mathrm{GeV}$ that give more asymmetric source $R_{O}^{*}=16.2 \mathrm{fm}, R=6.2 \mathrm{fm},\left\langle r^{*}\right\rangle=23 \mathrm{fm}$. The asymmetry of the sources leads to the deviation at LHC energies 5-6\% for kaons.

\section{Conclusions}

In this paper we have developed a practical approach to the size-dependent Coulomb corrections (instead of the simple zero-distance Coulomb factor) allowing one to extract the pure Bose-Einstein two-particle correlation functions of the bosons emitted from relatively large effective volumes.

It should be stressed that the complex behavior of the Coulomb effect with the increasing system size, generally, cannot be described in terms of a simple size-dependent Coulomb factor. In fact, for large effective sizes $\left\langle r^{*}\right\rangle$, constituting a substantial fraction of the pair Bohr radius $|a|$, the Coulomb effect becomes the main source of the space-time information on particle production. Then, it can no more be considered as a correction and has to be calculated numerically based on reasonable parameterization of the Wignerlike densities or on microscopic simulations using various transport codes. Nevertheless, we have shown that some useful analytical approximations for the size-dependent Coulomb factor are possible even for rather large systems.

The procedure of the extraction of the Bose-Einstein correlation function from the experimental correlation function of charged identical pions has been proposed. It is based on the use of $\pi^{+} \pi^{-}$correlation function and the formalism allowing to take into account the non-Gaussian sources as well as Gaussian ones.

This method cannot be applied for kaons due to the non-negligible con- 
tribution of the strong FSI for $K^{+} K^{-}$. Instead, using the facts of a relatively large kaon mass as compared with the pion mass and a moderate resonance contribution to the $K^{+} K^{+}$correlation function even at small $p_{T}$, the simple analytical approach for the thermalized 3D-expanding systems is proposed to estimate the contribution of the Coulomb FSI to the $K^{+} K^{+}$correlation function and determine the interferometric radii.

Acknowledgments: One of us (S.V.A.) gratefully acknowledges support by the Ukrainian State Fund of the Fundamental Research under Contract No 2.5.1/057.

\section{References}

[1] G.I. Kopylov, M.I. Podgoretsky, Sov. J. Nucl Phys. 15 (1972) 219; G.I. Kopylov, Phys. Letters B50 (1974) 472; M.I. Podgoretsky, Sov. J. Part. Nucl. 20 (1989) 266.

[2] G. Goldhaber, S. Goldhaber, W. Lee, A. Pais, Phys. Rev. 120 (1960) 325.

[3] Yu.M. Sinyukov, Nucl.Phys.A566 (1994) 589; Yu.M. Sinyukov, in: Hot Hadronic Matter: Theory and Experiment, eds. J. Letessier, H.H. Gutbrod and J. Rafelski (Plenum, New York, 1995) p. 309.

[4] Yu.M. Sinyukov, Nucl.Phys... A498 (1989) 151; S.V. Akkelin, Yu.M. Sinyukov, Phys LettersB356 (1995) 525; S.V.Akkelin, Yu.M.Sinyukov, Z.Phys.C72 (1996) 501.

[5] M. Gazdzicki et. al., NA35 Collaboration, Nucl. Phys. A590 (1995) 197c.

[6] L.D. Landau, Izv. Ac. Nauk USSR, Ser. Fis. 17 (1953) 51.

[7] J.D. Bjorken, Phys. Rev. D27 (1983) 140

[8] A.N. Makhlin, Yu.M. Sinyukov, Yad. Fiz. 46 (1987) 637; Z. Phys. C39 (1988) 69.

[9] K. Kolehmainen, M. Gyulassy, Phys. Letters B180 (1986) 203; S. Padula, M. Gyulassy, Nucl. Phys. B339 (1990) 378. 
[10] K. Kadija et. al., NA49 Collaboration, Nucl. Phys. A610 (1996) 248c; D. Ferenc, Nucl. Phys. A610 (1996) 523c.

[11] M. Gyulassy, S.K. Kauffmann, L.W. Wilson, Phys. Rev. C20 (1979) 2267.

[12] R. Lednicky, V. L. Lyuboshitz, Heavy Ion Phys. 3 (1996) 1.

[13] R. Lednicky, V. L. Lyuboshitz, Sov. J. Nucl Phys. 35 (1982) 770; R. Lednicky, V. L. Lyuboshitz, Proc. Int. Workshop on Particle Correlations and Interferometry in Nuclear Collisions, CORINNE 90, Nantes, France, 1990 (ed. D. Ardouin, World Scientific, 1990) p. 42.

[14] L.D. Landau, E.M. Lifshitz, Quantum Mechanics: Non-Relativistic Theory, 3-rd Ed.,Pergamon Press, Oxford, 1977.

[15] F.W.J. Olver, Asymptotics and Special Functions, Academic Press, New York, 1974.

[16] S. Pratt, Phys. Rev. D33 (1986) 72.

[17] M. Gmitro, J. Kvasil, R. Lednicky, V.L. Lyuboshitz, Czech. J. Phys. B36 (1986) 1281.

[18] M. Gyulassy, S.K. Kauffmann, Nucl. Phys. A362 (1981) 503; Y.D.Kim et. al., Phys.Rev. C45 (1992) 387; G.Baym, P.Braun-Munzinger, Nucl. Phys. A610 (1996) 286c.

[19] T. Alber et al., Z. Phys. C73 (1997) 443.

[20] M.Biyajima et. al., Phys. Lett. B353 (1995) 340; M.Biyajima et. al., Phys. Lett. B366 (1996) 394. 
RQMD, $\mathrm{Pb}-\mathrm{Pb}, 158 \mathrm{~A} \mathrm{GeV}$

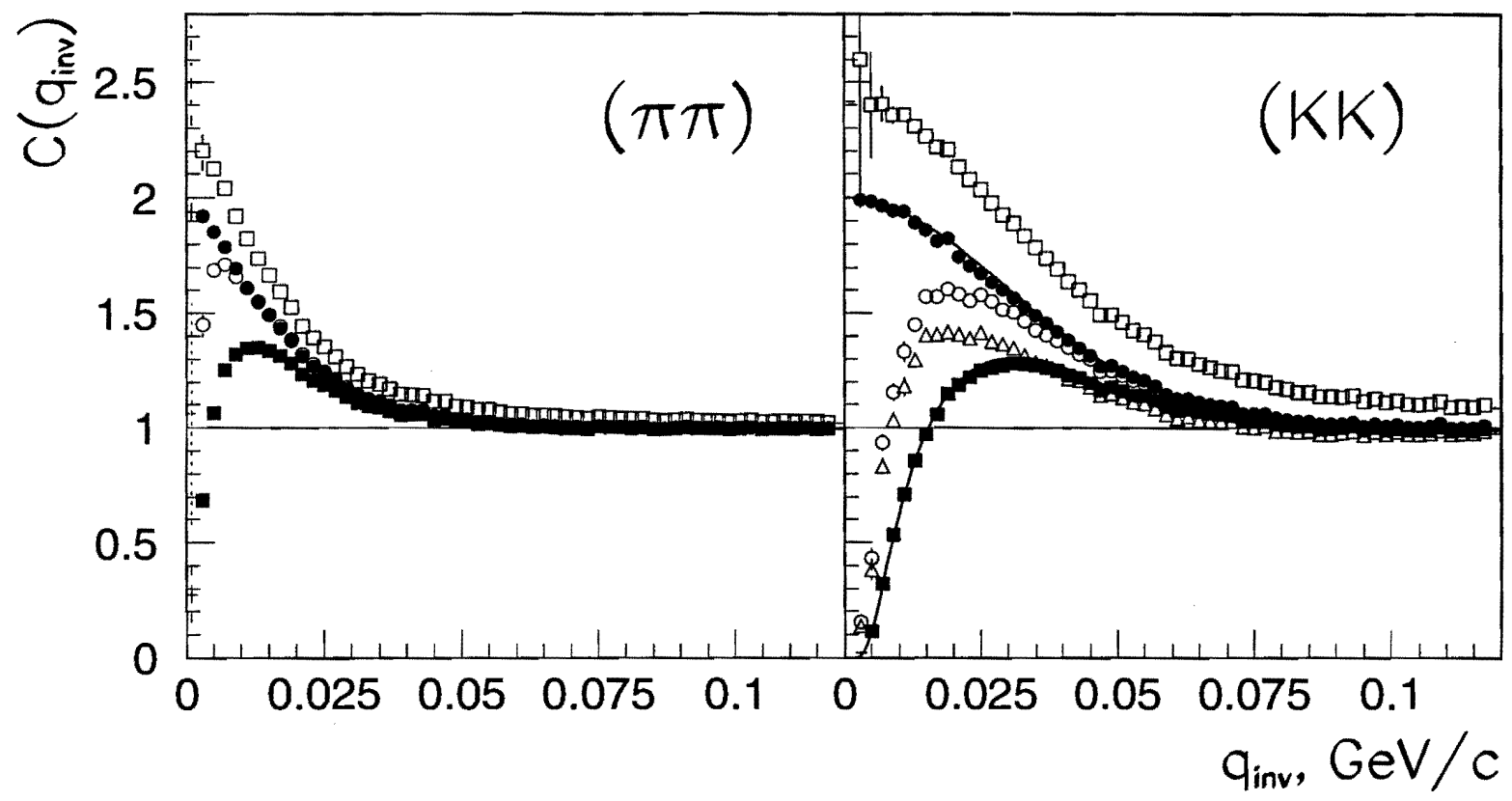

Fig.1. The correlations between charged bosons

- Complete calculation divided by Gamov factor

- Quantum statistics only

- Complete calc. multiplied by $\left(\pi^{+} \pi^{-}\right.$or $\left.\mathrm{K}^{+} K^{-}\right)$Coulomb c.f.

$\triangle$ Complete calculation multiplied by $\left(\mathrm{K}^{+} \mathrm{K}^{-}\right.$,complete) c.f.

- Complete calculation (QS+FSI)

The solid line corresponds to optimal combined fit for complete- and QS- correlation functions of $\mathrm{K}^{+} \mathrm{K}^{+}$pairs. 
$\pi^{+} \pi^{+}, \mathrm{RQMD}, \mathrm{Pb}+\mathrm{Pb} 158 \mathrm{~A} \mathrm{GeV}$

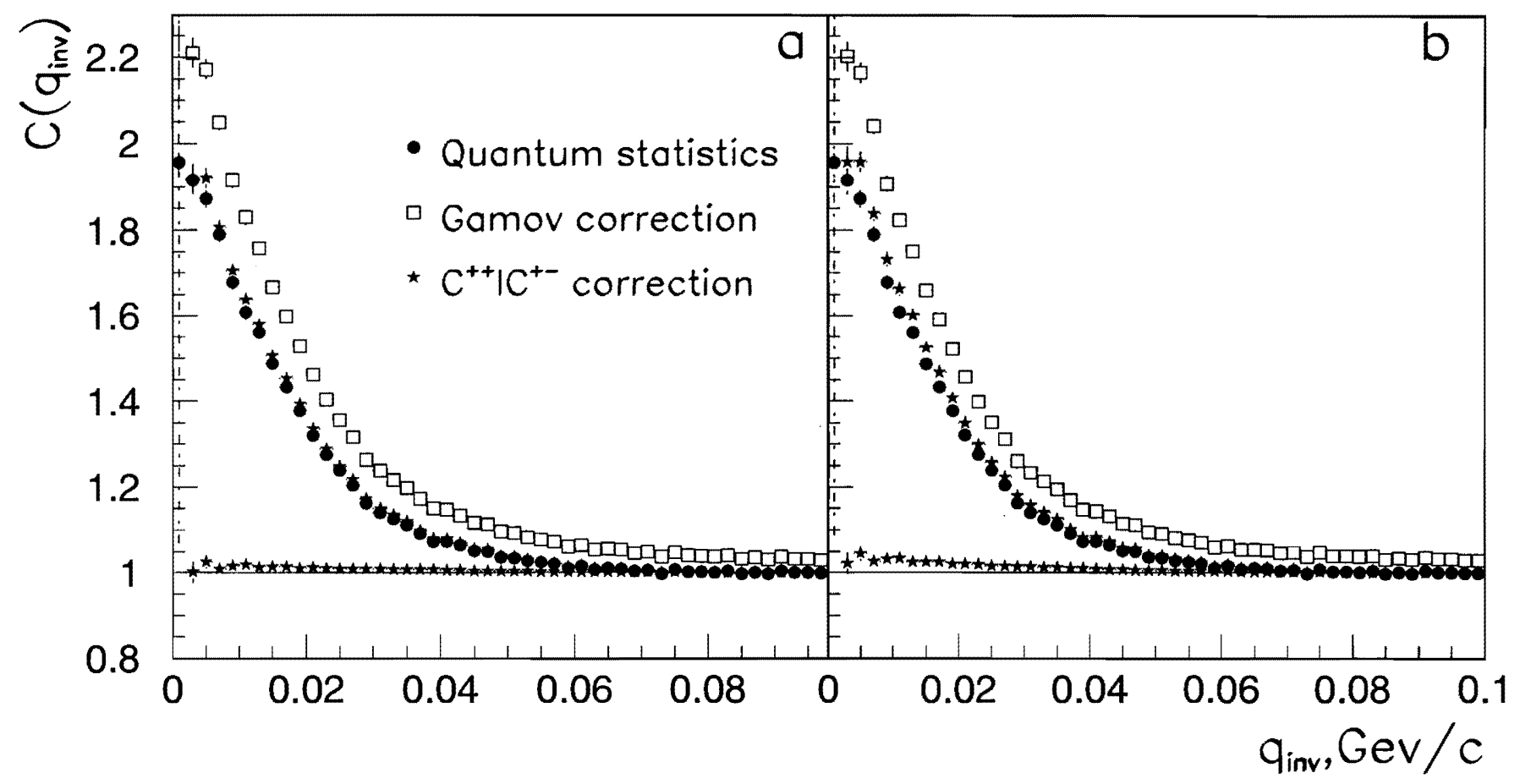

Fig.2. The comparison of the analytical approaches with Monte-Carlo simulations within RQMD model. $a$-without strong FSI, b-with it. 
$\pi^{+} \pi^{+}, \mathrm{HYDRO}, \mathrm{Pb}+\mathrm{Pb}$

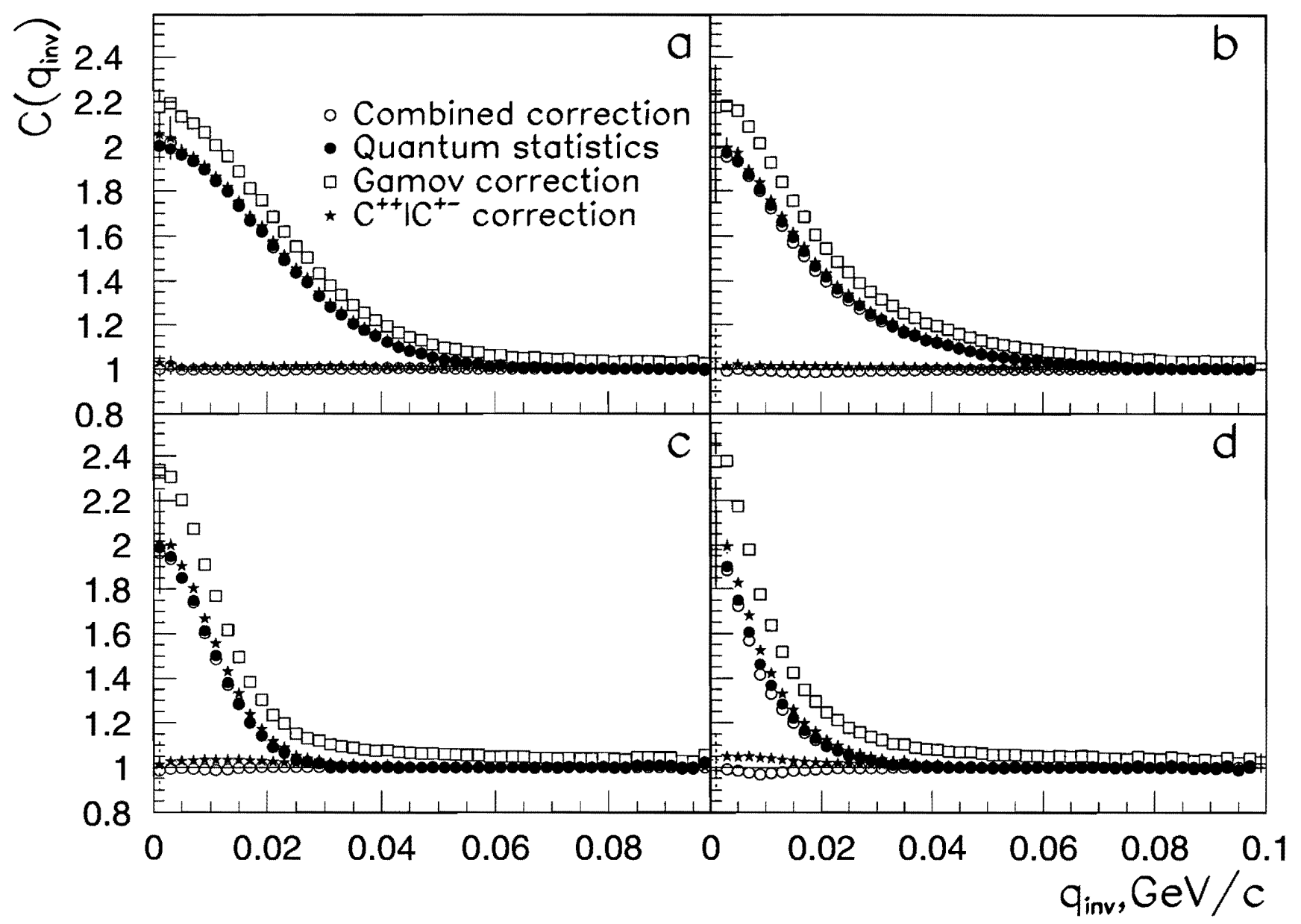

Fig.3. The comparison of the analytical approaches with HYDRO numerical calculations for SPS energy $(a, b)$ and $\mathrm{LHC}$ energy $(c, d) . a, c-p_{t}=0.15 \mathrm{GeV} / c, b, d-p_{t}=0.4 \mathrm{GeV} / c$. 
$\mathrm{K}^{+} \mathrm{K}^{+}, \mathrm{HYDRO}, \mathrm{Pb}+\mathrm{Pb}$

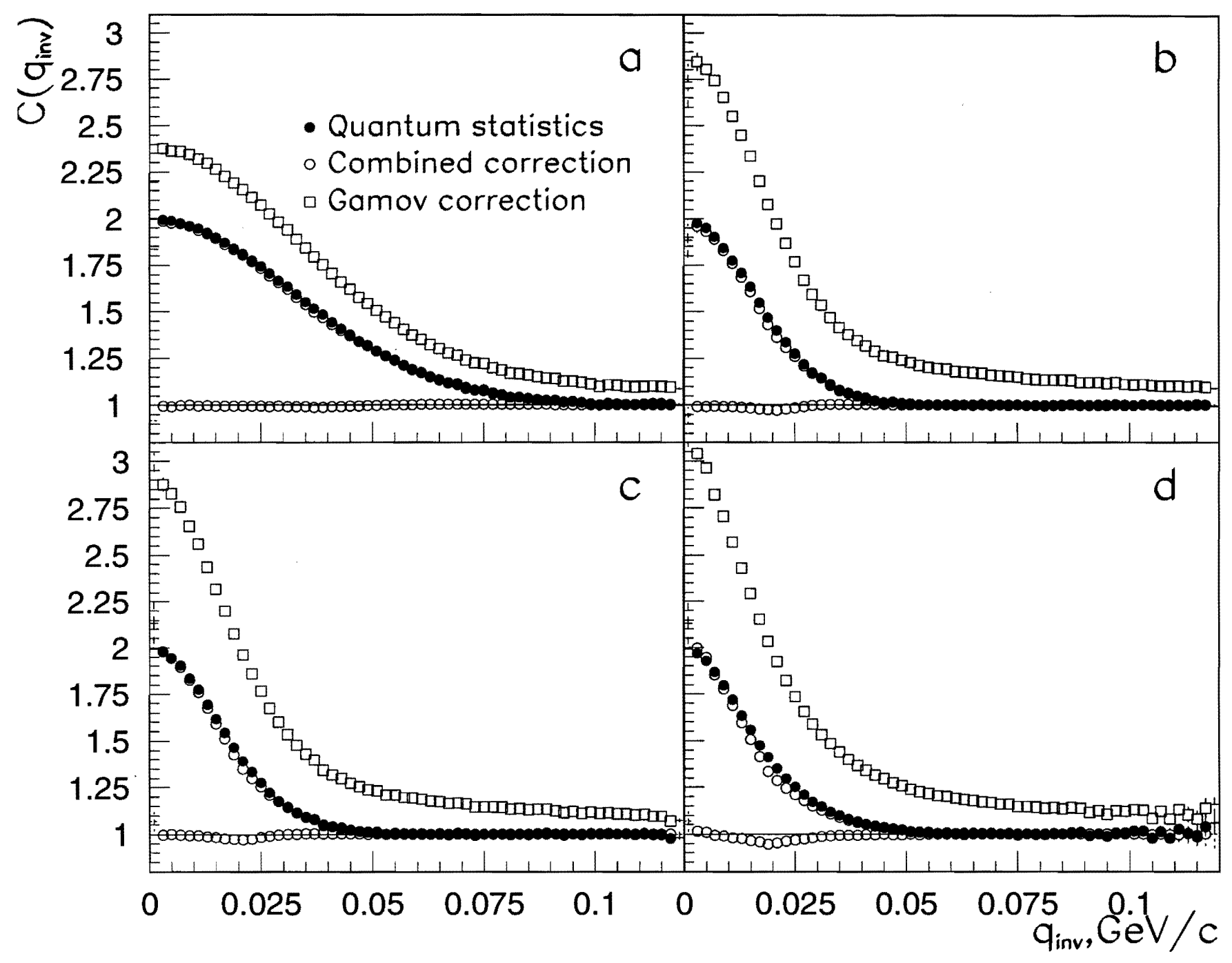

Fig.4. The comparison of the analytical approaches with HYDRO numerical calculations for SPS energy ( $a$ ) and LHC energy $(b, c, d), a, b: p_{t}=0.15 \mathrm{GeV} / c$, $c: p_{t}=0.4 \mathrm{GeV} / c, d: p_{t}=0.8 \mathrm{GeV} / c$. 